\title{
Treatment of severe hospital-acquired and ventilator-associated pneumonia: a systematic review of inclusion and judgment criteria used in randomized controlled trials
}

Emmanuel Weiss ${ }^{1,2,3^{*}}$, Wafa Essaied ${ }^{4}$, Christophe Adrie ${ }^{5}$, Jean-Ralph Zahar ${ }^{4,6}$ and Jean-François Timsit ${ }^{2,4,7}$

\begin{abstract}
Background: Hospital-acquired and ventilator-associated pneumonia (HAPNAP) are often selected for randomized clinical trials (RCTs) aiming at new drug approval. Guidelines for the design of such RCTs have been repeatedly updated by regulatory agencies. We hypothesized that large variability in the enrolled populations, the endpoints assessed and the HAPNAP definition criteria may impact the results of these studies, and addressed this through a systematic review of HAPNAP RCTs.
\end{abstract}

Methods: A search (Pubmed-Embase-ICAAC-ECCMID) of all RCTs published between 1994 and 2016 comparing antimicrobial treatment for HAPNAP in the intensive care unit was conducted. The populations enrolled, inclusion/ exclusion criteria, statistical design and endpoints assessed were recorded. All unpublished RCTs recorded on the ClinicalTrials.gov registry were also screened.

Results: From the 93 abstracts reviewed, 39 potentially relevant studies were inspected, leading to 27 studies being included. As expected, illness severity or the proportion with VAP (27-100\%) differed greatly among the enrolled populations. The HAPNAP definition used various clinical and biological criteria, and only 55\% of studies required a microbiological sample. The mandatory duration of prior hospital stay was variable; the mechanical ventilation duration was an inclusion criterion in only $41 \%$ of VAP studies. Nine studies had non-inferiority design, but nine studies (33\%) did not have a pre-specified statistical hypothesis. Clinical cure was the primary endpoint in 24 studies, but was recorded in several populations or as the co-primary endpoint in 13 studies. The definition of clinical cure and the timing of its assessment greatly differed. This variability slightly improved over time but remained significant in the 13 registered but currently unpublished RCTs that we screened.

Conclusion: Our study provides a description of populations and endpoints of RCTs evaluating antimicrobials for treatment of HAPNAP in the ICU. There was significant heterogeneity in enrollment criteria, endpoints and statistical design, which may influence the ability of studies to demonstrate differences between studied drugs.

Keywords: Systematic review, Hospital-acquired pneumonia treatment, Ventilator-acquired pneumonia treatment, Randomized controlled trials, Endpoints, Inclusion criteria

\footnotetext{
* Correspondence: emmanuel.weiss@aphp.fr

${ }^{1}$ Department of Anesthesiology and Critical Care, HUPNVS, Hôpital Beaujon, APHP, 92110 Clichy, France

${ }^{2}$ Paris Diderot University, Paris, France

Full list of author information is available at the end of the article
} 


\section{Background}

New antimicrobials are required to face the dramatically increasing prevalence of multidrug-resistant pathogens. This unmet need has become a worldwide source of concern, and government action plans aiming at increasing the antimicrobials pipeline are in development [1]. The evaluation of these new treatments will require properly designed studies with appropriate inclusion criteria and endpoints. However, in hospital-acquired pneumonia (HAP) and ventilator-associated pneumonia (VAP), the way to conduct such studies remains unclear. During the past ten years, the regulatory agencies (European Medicines Agency (EMA) and Food and Drug Administration (FDA)) have repeatedly updated their guidelines for the design of randomized controlled trials (RCTs), but their recommendations remain conflicting, especially regarding the design, endpoints, or inclusion criteria that should be used $[2,3]$.

This is an important issue because HAP and VAP are ideal syndromes for clinical trials in the context of new drug approval: they are a major cause of infection, often involving multidrug-resistant pathogens ( $25 \%$ of ICU infections), they account for up to $50 \%$ of antibiotic prescriptions [4], identification of the causative pathogen using microbiological samples is easy, and they are particularly sensitive to antimicrobial treatment effect $[5,6]$.

Our hypothesis is that to date, HAP/VAP RCTs greatly differ in the population enrolled, the criteria used for definition of HAP/VAP, and the endpoints assessed. Such differences among studies may be of importance as they may impact the results of the studies. For example, some studies showed that the reported incidence of VAP greatly depends on the diagnostic methodology used. This systematic review describes the enrolled populations, design, and conduct of RCTs addressing the efficacy of antimicrobials for HAP/VAP treatment in intensive care unit (ICU) patients from 1994 to 2016.

\section{Methods}

The methodology of the Preferred Reporting Items for Systematic Reviews and Meta Analyses (PRISMA) guidelines was used for the conduct of the systematic review [7].

\section{Search Strategy}

We searched PubMed, Embase, ICAAC and ECCMID for publications from 1994 to 2016. The search string used was:

1-(Pneumonia and (hospitalized or acquired or ventilatoryassociated or nosocomial or health-associated)) and (antibiotic treatment or (antibiotic therapy) or antibiotic or (antibacterial agents) or (anti-infective agents) or antimicrobial)

It was complemented by:
2-Pneumonia and (hospitalized or acquired or ventilatoryassociated or nosocomial or health-associated)) and (doripenem or meropenem or impenem or linezolid or vancomycin or piperacillin or (piperacillin and tazobactam) or ceftazidim or ceftriaxone or cefotaxime or ceftobiprole or ciprofloxacin or levofloxacin or moxifloxacin or piperacillin or teicoplanin or cefepime or ticarcillin or (ticarcillin and clavulanic acid) or amikacin or tobramycin or gentamicin) and (randomized controlled trial).

Of note, some studies were identified through sources other than a database search, including contact with researchers.

\section{Study selection}

All RCTs evaluating the efficacy of antimicrobials in HAP/ VAP in adult patients in the ICU from 1994 to 2016 were considered as eligible for this systematic review. The study selection process is detailed in a flow diagram (Fig. 1). The search period was limited to twenty years because of the evolution of publication requirements and treatment concepts. All articles were independently screened by two authors (J-RZ and CA). Full texts (and clinical trial registry data (ClinicalTrials.gov website)) of all potentially relevant studies were analyzed to assess eligibility. Disagreements on the inclusion/exclusion of studies were resolved in consultation with other co-authors (EW and J-FT). We included studies comparing one antibiotic agent to another, monotherapy to combination therapy, inhaled to systemic route or extended/continuous infusion to intermittent administration. Finally, in January 2017 we also searched the ClinicalTrials.gov website for characteristics of currently unpublished RCTs comparing HAP/VAP antimicrobial treatment strategies.

\section{Recorded data}

The following data were collected for each study included: inclusion and exclusion criteria, design, primary endpoint and population in which it was analyzed, definition of clinical cure, time point used for the test of cure (TOC) visit, and the statistical hypothesis. Data extraction from the studies was confirmed by an independent reviewer. At the end, a global quality assessment of each RCT was performed using an 8-item scale allowing calculation of a global quality score adapted to the focus of this systematic review.

\section{Results \\ Populations enrolled}

The main characteristics of the 27 studies included in the systematic review (see Additional file 1 for references) and of their enrolled populations are described in Table 1.

The number of patients enrolled in the intention-totreat (ITT) population was highly variable and ranged from 35 to 945 . Patients were predominantly male and 


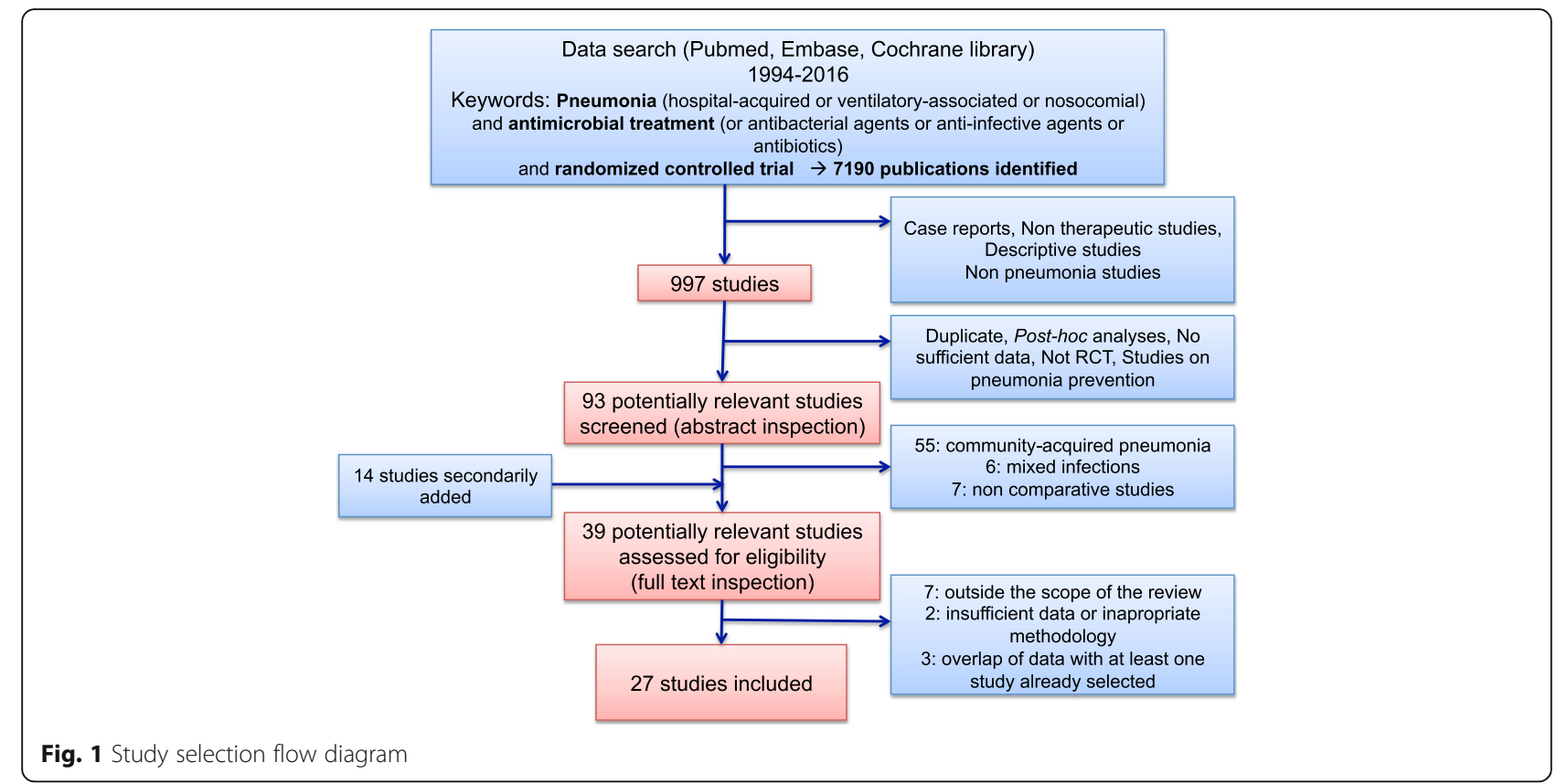

the mean age across trials was $50.5-70.0$ years. In most studies (92\%), severity of illness was reported using the APACHE II score and its mean value varied from 13.8 to 24.0 across trials. The rate of comorbidity was rarely reported.

Sixteen studies co-enrolled patients with HAP and VAP and 11 studies exclusively enrolled patients with VAP. Among the former, the proportion of patients with VAP greatly varied from 27 to $91 \%$. In some studies, randomization was stratified by patients' severity of illness (three trials) and on the rate of VAP (two trials). Whereas 18 studies (67\%) included all HAP/VAP episodes regardless of the causative pathogen, some only enrolled patients with HAP/VAP related to specific pathogens. The duration of hospital stay before the onset of pneumonia was required to be longer than 48 hours and 72 hours in 18 studies (67\%) and 7 studies (26\%), respectively (not mentioned in the remaining study). However, the duration of the mechanical ventilation was an inclusion criterion in only 11 VAP studies (41\%).

The diagnosis of pneumonia was consistently based on clinical and radiological (new lung infiltrate on chest radiography) findings but microbiological confirmation was required in only 15 studies (55\%). Among the remaining studies, the proportion of patients analyzed who had positive respiratory samples varied from 62 to $82 \%$. Clinical signs leading to suspicion of HAP/VAP were highly variable across the 27 studies. These clinical findings and the percentage of studies using them as inclusion criteria are shown in Fig. 2a. In $73 \%$ of studies, a predefined number of respiratory symptoms and signs of sepsis were required for pneumonia diagnosis and subsequent inclusion (Fig. 2b).
Various exclusion criteria were also reviewed. In five studies (20\%), patients with severe disease, as reflected by high Acute Physiology and Chronic Health Evaluation (APACHE) score (four studies) or Simplified Acute Physiology Score (SAPS) (one study), concomitant septic shock (two studies) or acute respiratory distress syndrome (one study) were excluded. Neutropenia and immunosuppression (various definitions) were considered as exclusion criteria in 13 studies (48\%) and 7 studies (26\%), respectively. Finally, patients with chronic respiratory diseases were excluded in nine studies (33\%).

Only a few studies tried to balance heterogeneity by stratifying the randomization according to the severity of the acute disease (APACHE in two studies and partial arterial oxygen pressure/fraction of inspired oxygen $(\mathrm{PaO} 2 / \mathrm{FiO} 2)$ ratio in one study) or the characteristics of the pneumonia (i.e., HAP or VAP, in three studies).

\section{Clinical trial design}

There were 21 multicenter studies (78\%), 14 doubleblinded studies (52\%) and 13 open-labeled studies (48\%). The duration of study therapy varied from one study to another (from 5 to 21 days) and was left at the discretion of the investigators in $37 \%$ of studies.

Nine trials (33\%) used a non-inferiority design with non-inferiority margins (i.e., absolute percentage difference in the primary outcome acceptable for non-inferiority to be established) mentioned in eight of them $(20 \%, 15 \%$ and $10 \%$ in three studies, four studies, and one study, respectively), but the scientific evidence leading to calculation of the non-inferiority margin was missing in two of them. Six studies (22\%) and three studies (11\%) were equivalence 


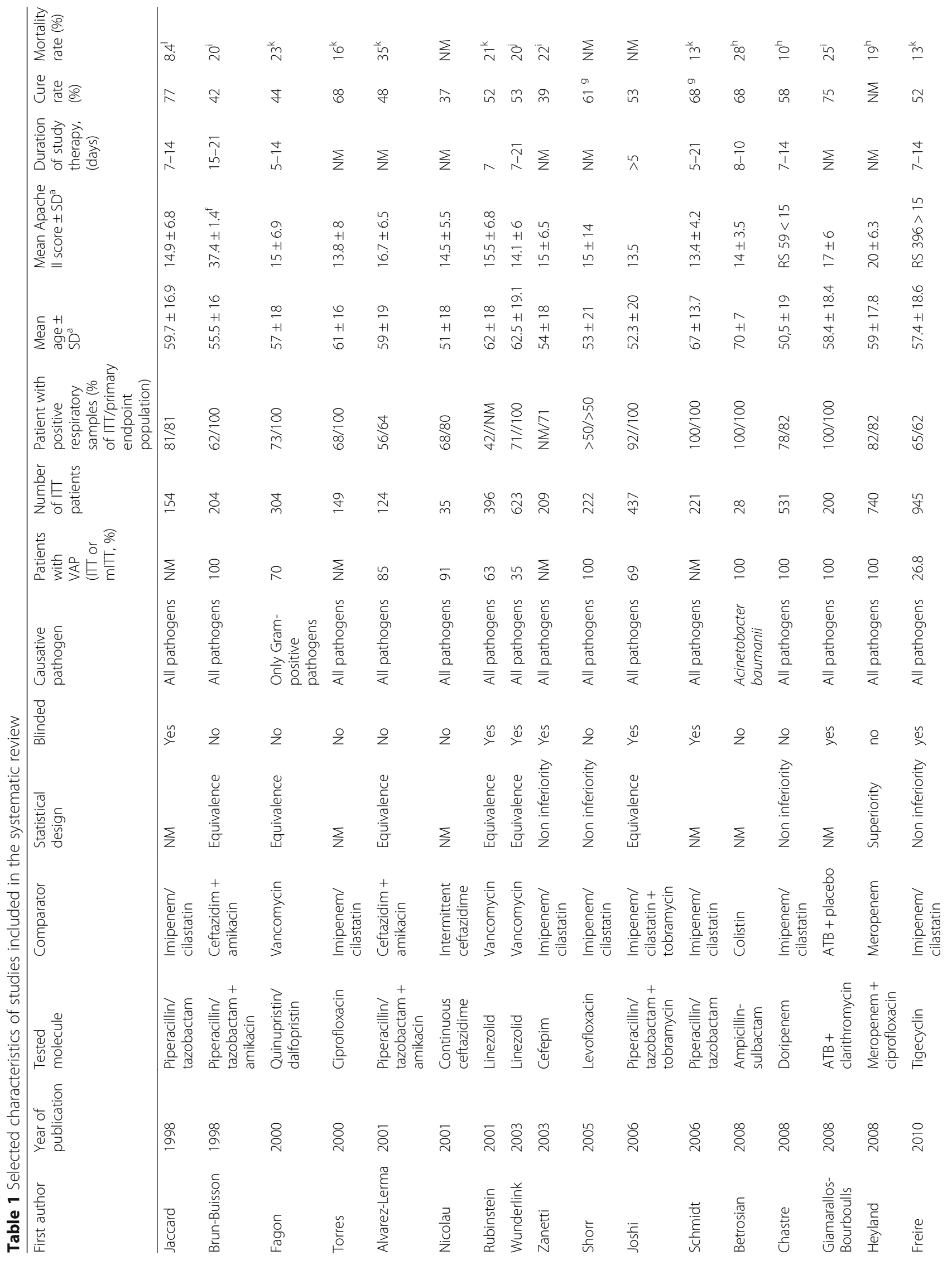




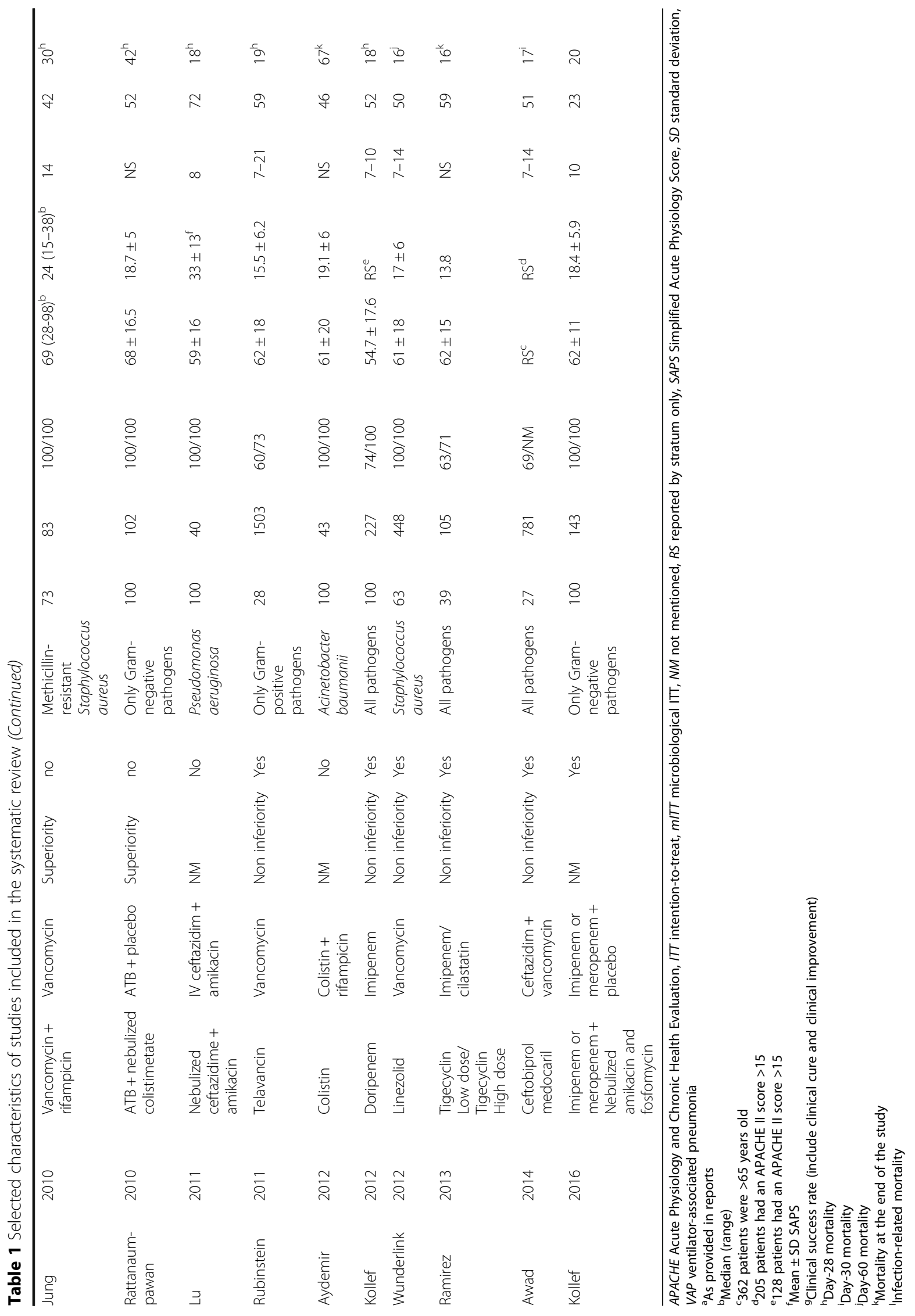


a Signs and symptoms used for HAP/NAP diagnosis $(\mathrm{n}=27)$

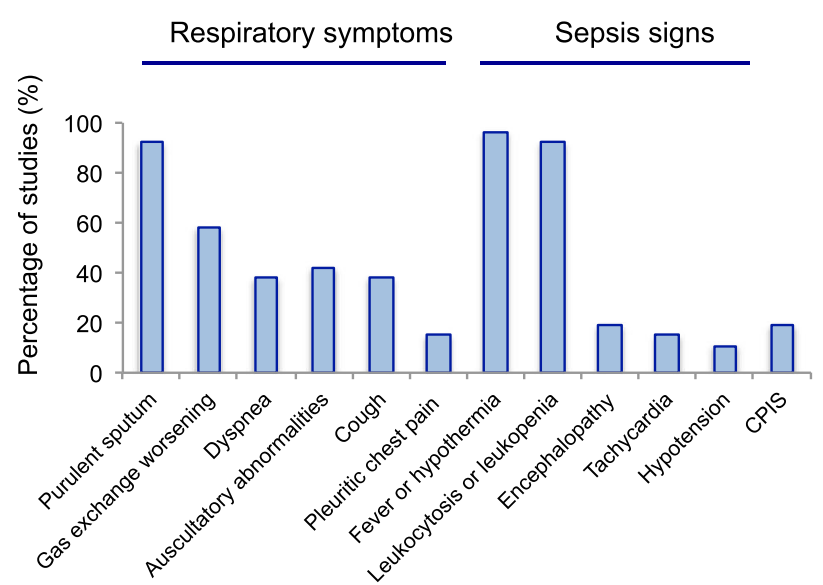

b Number of signs and symptoms required for HAPI VAP diagnosis

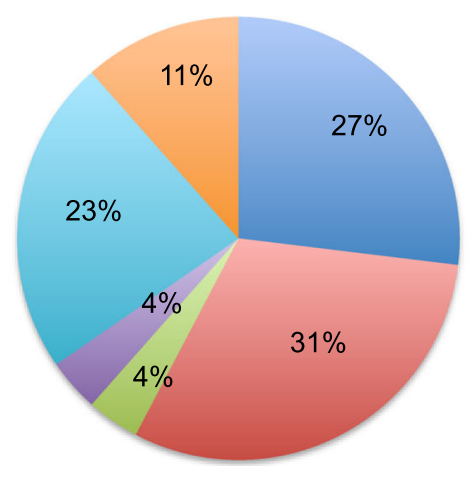

Not mentionned

च2 signs (respiratory or sepsis)

$\geq 3$ signs (respiratory or sepsis)

1 sepsis sign

a1 sepsis sign and $\geq 2$ respiratory signs

\pm 2 septic signs and $\geq 2$ respiratory signs

Fig. 2 Variability of signs and symptoms used for definition of hospital-acquired pneumonia/ventilator-associated pneumonia (HAPNAP) ( $n=27$ studies). a Frequency of signs and symptoms used as HAPNAP diagnostic criteria. b Number of signs and symptoms required for HAPNAP diagnosis. CPIS Clinical Pulmonary Infection Score

and superiority trials, respectively. Of note, a pre-specified statistical hypothesis was lacking in nine studies $(33 \%)$ and sample size calculation was not mentioned in nine studies (33\%).

\section{Primary endpoint}

Our work revealed marked heterogeneity among the primary endpoints reported in HAP/VAP trials. A single primary endpoint in one population was reported in 13 studies (48\%): a clinical cure in 12 trials (44\%), CPIS decrease in 1 trial (4\%), and 28-day mortality in 1 (4\%).
In seven studies, the clinical cure was analyzed in several populations (two populations in six studies, and three populations in one study) without specifying which one was the primary endpoint. In four studies, the clinical cure and microbiological cure were both reported as the primary endpoint and safety was also added in two studies. Altogether, clinical cure was the primary endpoint (or one of the "primary" endpoints) in 24 studies (89\%) and mortality in only 2 studies. The various populations used to analyze clinical cure are displayed in Fig. 3a.

\section{a Populations in whom clinical cure was analyzed}

\section{b}

Elements included in cure definition
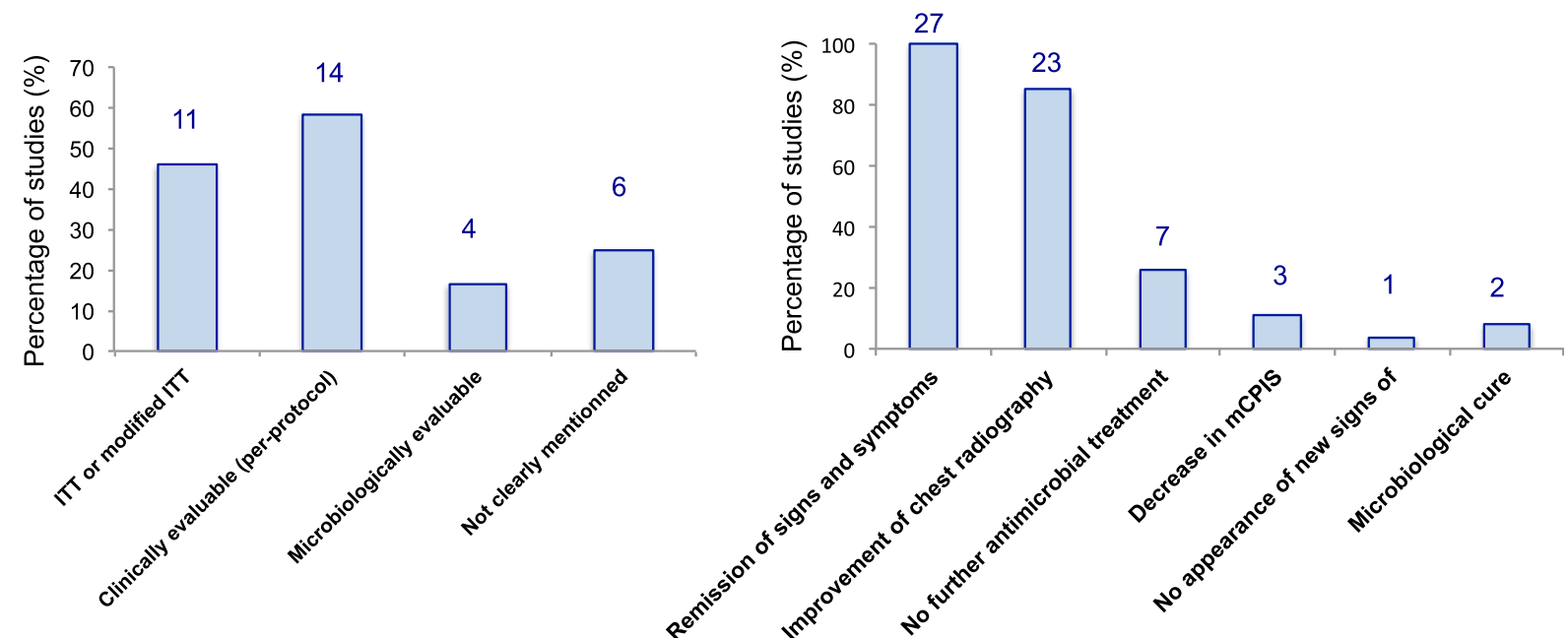

Fig. 3 Clinical cure analysis. a Populations in whom clinical cure was analyzed $(n=24$ studies. $/ \pi$ intention-to-treat. $\mathbf{b}$ Items included in the definition of clinical cure. mCPIS modified Clinical Pulmonary Infection Score 
For a global assessment of study quality, we calculated an 8-point quality score based on 8 systematically evaluated items, reflecting enrollment criteria and methodology (Table 2). Altogether, this score ranged from 2 to 7 , thereby confirming the heterogeneity between studies.

\section{Definition of clinical cure and timing of the assessment}

Interestingly, elements included in the definition of clinical cure greatly differed among studies (Fig. 3b). Remission of signs and symptoms of pneumonia were included in the definition of clinical cure in all studies, but was only partial in $11(41 \%)$ studies. Chest radiography findings were not systematically taken into account $(22$ studies, $82 \%$ ) and they qualified for clinical cure whether the improvement was complete, partial or only a lack of progression in 18 studies (67\%). Finally, no need for additional antibiotics during follow up and decrease in the Clinical Pulmonary Infection Score (CPIS) [8] were used in $26 \%$ and $11 \%$ of studies, respectively. The timing of the TOC visit was different across trials. The time point was between day 7 and day 21 after the end of therapy in $56 \%$ percent of the studies; in the remaining trials, it ranged from the end of treatment (4 studies, 15\%) till day 30 after treatment (3 studies, 12\%). These differences in the definition of clinical cure and in the population and time points used to assess it led to wide variability in reported clinical cure rates that ranged from 23 to $77 \%$ (Table 1).

Table 2 Quality assessment of the included randomized controlled trials using eight items and allowing calculation of a quality score

\begin{tabular}{|c|c|c|c|c|c|c|c|c|c|c|}
\hline & & \multicolumn{3}{|c|}{ Enrolment criteria } & \multicolumn{5}{|c|}{ Methodological quality } & \multirow[b]{2}{*}{$\begin{array}{l}\text { Quality } \\
\text { score }\end{array}$} \\
\hline First author & Date & $\begin{array}{c}\text { Severity of the } \\
\text { population } \\
\text { (mean APACHE } \\
\text { II >15) }\end{array}$ & \begin{tabular}{|c|} 
Proportion of \\
patients with \\
VAP mentioned
\end{tabular} & $\begin{array}{c}\text { Microbiological } \\
\text { confirmation of } \\
\text { HAP/NAP } \\
\text { mandatory }\end{array}$ & Blindness & $\begin{array}{c}\text { Clear statistical } \\
\text { design }\end{array}$ & $\begin{array}{c}\text { One single } \\
\text { endpoint }\end{array}$ & \begin{tabular}{|c|} 
One single \\
population of interest \\
as primary endpoint \\
\end{tabular} & \begin{tabular}{|c} 
Clear \\
statistical \\
design
\end{tabular} & \\
\hline Jaccard & 1998 & & & & & & & 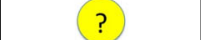 & & 3 \\
\hline Brun-Buisson & 1998 & & & & & & & & & 5 \\
\hline Fagon & 2000 & & & & & & & & & 5 \\
\hline Torres & 2000 & & & & & & & & & 3 \\
\hline Alvarez-Lerma & 2001 & & & & & & & & & 3 \\
\hline Nicolau & 2001 & & & & & & & & & 2 \\
\hline Rubinstein & 2001 & & & ? & & & & & & 5 \\
\hline Wunderlink & 2003 & & & & & & & & & 5 \\
\hline Zanetti & 2003 & & & & & & & & & 2 \\
\hline Shorr & 2005 & & & & & & & & & 3 \\
\hline Joshi & 2006 & & & & & & & & & 6 \\
\hline Schmidt & 2006 & & & & & & & & & 5 \\
\hline Betrosian & 2008 & & & & & & & & & 4 \\
\hline Chastre & 2008 & & & & & & & & & 4 \\
\hline Giamarallos & 2008 & & & & & & & & & 4 \\
\hline Heyland & 2008 & & & & & & & & & 5 \\
\hline Freire & 2010 & & & & & & & & & 4 \\
\hline Jung & 2010 & & & & & & & & & 4 \\
\hline Rattanaumpawan & 2010 & & & & & & & & & 3 \\
\hline Lu & 2011 & & & & & & & & & 3 \\
\hline Rubinstein & 2011 & & & & & & & & & 4 \\
\hline Aydemir & 2012 & & & & & & & & & 4 \\
\hline Kollef & 2012 & & & & & & & & & 6 \\
\hline Wunderlink & 2012 & & & & & & & & & 8 \\
\hline Ramirez & 2013 & & & & & & & & & 5 \\
\hline Awad & 2014 & & & ? & & & & & & 4 \\
\hline Kollef & 2016 & & & & & & & & & 6 \\
\hline
\end{tabular}




\section{Mortality}

All-cause mortality was reported in 22 studies (81\%) (primary endpoint in one study) and HAP/VAP-related mortality was reported in 2 studies (Table 1). The time points used to assess mortality were highly variable: day 28 in nine studies, at the end of the study in eight studies, and day 30 and day 60 in four studies and two studies, respectively. At day 28 , the mean mortality rate was $23 \%$, with broad variability of the reported allcause mortality rates from one study to another (range 10-67\%; Table 1).

\section{Improvement in methodological quality over time}

The methodological quality of studies improved over time. We specifically analyzed the six studies published after the EMA workshop on antimicrobials held in 2011, i.e., between 2012 and 2016. Among the six studies, four had a non-inferiority design (with anticipated statistical approach and non-inferiority margin), were doubleblinded, and assessed clinical cure in one population as the single primary endpoint. Clinical criteria for diagnosis of HAP/VAP were more homogenous and at least two clinical signs were consistently required. Furthermore, all cases of pneumonia that were analyzed were microbiologically proven in four of the six trials. Finally, the only items that did not improve were the definition of clinical cure and the time points used to assess it. Calculation of the mean quality score confirmed this global improvement: the mean score of studies published after 2011 was higher than that of older studies (5.3 vs 3.9) (Table 2).

\section{Features of currently unpublished RCTs reported on ClinicalTrials.gov. comparing antimicrobial treatment strategies in HAP/VAP}

Thirteen currently unpublished RCTs that tested new antimicrobial treatment strategies in patients with HAP/ VAP or with severe infections due to carbapenemresistant bacteria including $\mathrm{HAP} / \mathrm{VAP}$ are registered on ClinicalTrials.gov (Table 3). Interestingly, the primary endpoint remained highly variable: 28 -day all-cause mortality in four studies (all comparing systemic molecules) and clinical cure (with a variable definition, and using variable timing of assessment) in nine studies (four of them comparing systemic molecules and five testing the efficacy of nebulized antimicrobials) and a favorable clinical response. Only five studies described which population was analyzed.

\section{Discussion}

\section{Main findings}

In this systematic review comprising 27 published RCTs addressing the efficacy of antimicrobials for treatment of HAP/VAP in critically ill patients, we found that the enrolled populations, clinical trial design, and endpoints assessed vary greatly between studies. This variability is intuitive but its quantification is of importance because, as shown by the variable mortality and clinical cure rates that were reported, it impacted the results of RCTs. This heterogeneity may therefore influence the ability of studies to demonstrate differences between investigational drugs and comparators. These results are not surprising as far as there are even differences in the guidance from regulatory agencies on how to evaluate treatments for HAP/VAP [2, 3], especially with regards to the study design, population and endpoint.

\section{Enrolled populations}

In parallel with the inconsistent recommendations of the regulatory agencies on methodology for the diagnosis of $\mathrm{HAP} / \mathrm{VAP}$, we found great variability in the clinical criteria used for clinical diagnosis of HAP/VAP. These discrepancies were probably increased by the co-enrollment of patients with HAP and VAP in the majority of studies. Indeed, in agreement with the FDA, but not with the EMA guidelines, no distinction was made between clinical signs and symptoms used for the diagnosis of these different diseases; a minimal duration of mechanical ventilation was required as an inclusion criterion in only $41 \%$ of studies. Such a merge of populations with HAP and VAP could mask differences between groups in drug activity, and lead to false conclusions [9]. According to several authors, a combination of clinical and radiological criteria should be used to increase the pretest probability of disease before confirmatory microbiology culture for diagnosis of HAP and VAP $[8,10]$. However, in our review, the number of required clinical and biological symptoms greatly varied, and scores combining clinical, biological, and microbiological data such as CPIS [8] were almost never used.

Similarly, in the context of HAP/VAP treatment trials, microbiological confirmation of pneumonia is left optional by the EMA; it was used as inclusion criterion in only $55 \%$ of studies included in our review, and among the remaining studies the proportion of microbiologically documented pneumonia varied between 60 and $80 \%$. As a consequence, some patients without pneumonia may have been enrolled in some studies and may have led to false negative results in superiority trials and false conclusions of similarity of drugs in non-inferiority trials. Finally, the severity scores of enrolled patients were very different from one study to another and, in some studies the most severe patients were excluded. The lack of inclusion of some subgroups of patients may be questionable as far as they may specifically receive the maximum benefit of the tested antibiotic $[11,12]$. Stratification of randomization of patients based on the severity of illness unfortunately was rarely used. 


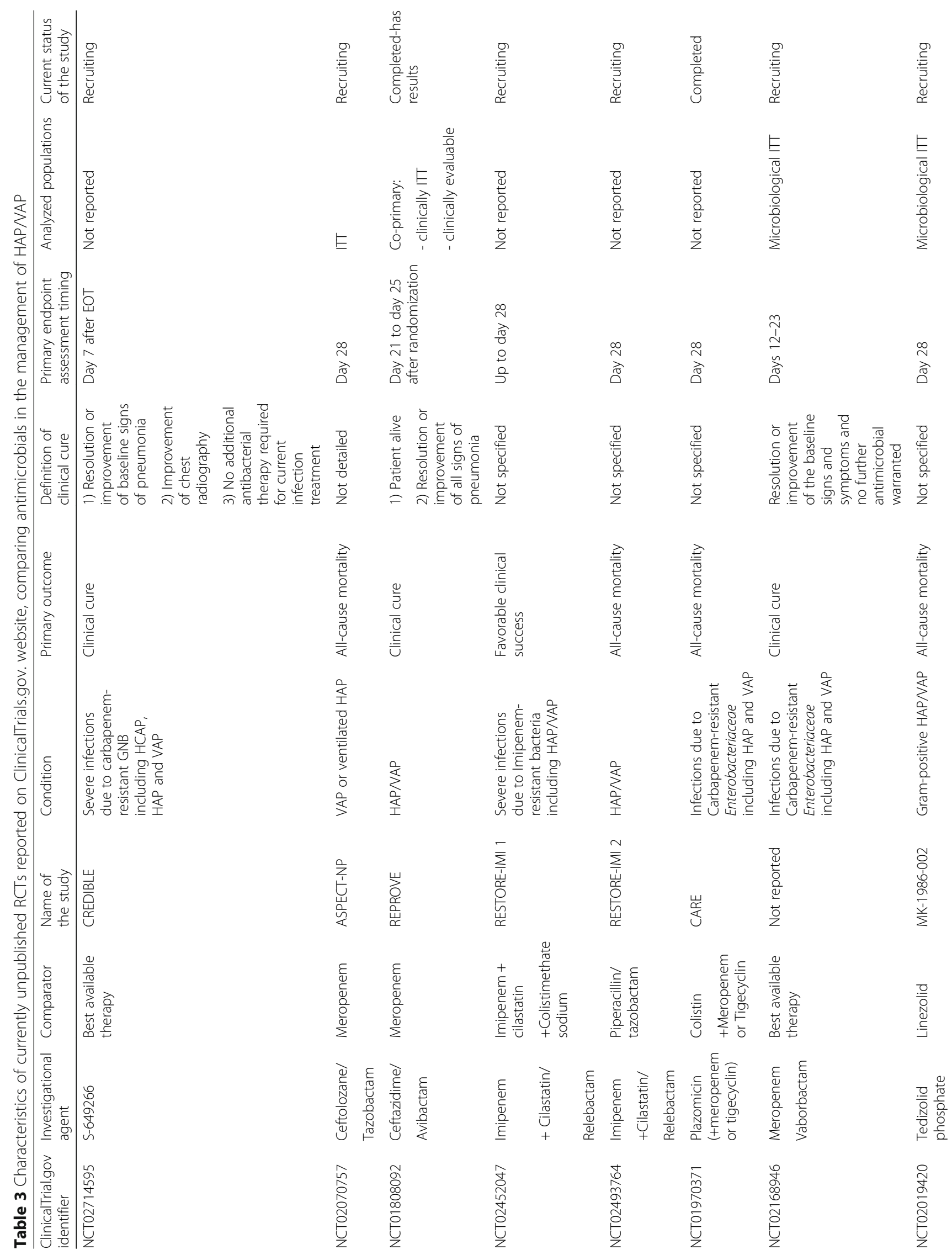




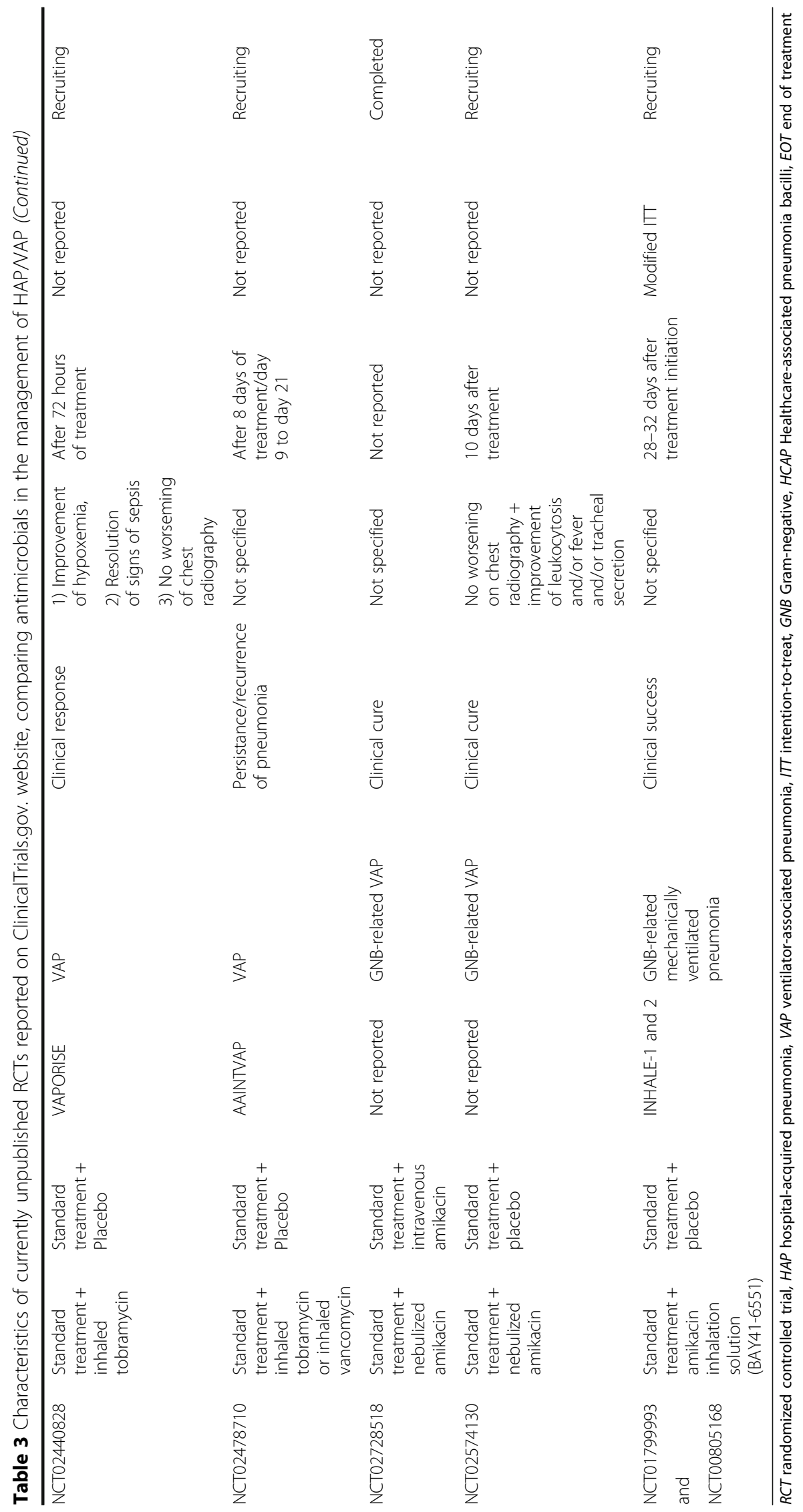




\section{Design}

We identified a large proportion of studies with methodological issues. Although drug dose monitoring or use of multiple antibacterial agents may complicate doubleblinding in HAP/VAP studies, it remains crucial to minimize post-randomization bias. In our review, doubleblinding was performed in only half of the studies. Furthermore, a pre-specified statistical hypothesis was lacking in more than one study out of four, and the sample size calculation was not mentioned in more than one out of three. Of note, only three studies had superiority design. A statistical hypothesis of non-inferiority was three times more frequent and increased over time (four out of the five most recent studies used it). The use of this design may be the result of FDA and EMA recommendations that describe this design as "acceptable" $[2,3]$. Nevertheless, non-inferiority trials are complex to conduct. They require an appropriately selected narrow non-inferiority margin and consequently a large sample size to prevent false conclusions of non-inferiority of the tested drugs. In this context, according to the FDA, mortality noninferiority margins should be less than 10\% [2]. According to the EMA, clinical cure non-inferiority margins should be less than $12.5 \%$ [3]. In our study, clinical cure noninferiority margins were frequently mentioned but they were larger than those recommended in almost all studies, which is clinically unacceptable.

\section{Endpoints}

The question of the best primary efficacy endpoint to use in HAP/VAP trials remains highly debatable [13]. Proof of this is provided by the conflicting guidelines provided by the regulatory agencies, with the EMA recommending the clinical outcome at the TOC visit (ranging from 14 to 21 days after the end of therapy) and the FDA recommending all-cause mortality at day 28 [2].

Our results show that the assessment of clinical response (clinical cure) by investigators was used in the vast majority of HAP/VAP RCTs. The main advantage of this criterion is its routine use by clinicians to assess patient response to an antimicrobial treatment. However, as demonstrated by the wide range of clinical cure rates showed in this review, the variable definitions of clinical cure that are used are problematic and may impact the reliability of this endpoint. These results suggest that a consensual definition of clinical cure is urgently needed. Such consensus should also homogenize the timing used to assess the primary endpoint, in order to limit its variability that may also account for variable clinical cure rates. In our review, whatever the endpoint used (i.e., clinical cure or mortality), there was wide variability in the time point used to assess it. Finally, the documentation of the magnitude of treatment effect on clinical cure should also be established. Indeed, a previous demonstration of the superiority of the comparator drug to a placebo or no therapy is necessary before conducting non-inferiority trials [14], and in HAP/VAP such benefit of effective antibacterial therapy has only been established for all-cause mortality [15] and not for clinical cure.

For this reason, mortality is the only endpoint that should be used in non-inferiority trials. However, while mortality is the endpoint that reflects the strongest outcome criteria, its choice may also offer some disadvantages. First, the mortality attributable is difficult to determine; allcause mortality may be related to underlying comorbidity, affecting the relationship between the efficiency of the antimicrobial treatment and death. Second, low mortality rates reported in HAP/VAP trials (except for those enrolling patients with difficult-to-treat pathogens) and the large non-inferiority margins that are recommended may prevent rejection of the non-inferiority of the tested drug and biased the results.

Nevertheless, the latest FDA recommendations [2] may promote the use of mortality as a primary endpoint. Indeed, among the five currently unpublished RCTs of new systemic molecules that are reported on the ClinicalTrials.gov website, two use 28-day all-cause mortality as the primary endpoint, and one includes death in the definition of clinical cure. Clinical cure was used in seven studies, including one addressing the effect of inhaled antimicrobials as adjunctive therapy. As expected, as a clear consensus is still lacking, the variability in the definition of clinical cure and in the time point used to assess it remain significant.

\section{Conclusion}

Altogether, this review provides a description of populations and endpoints of RCTs evaluating antimicrobials for treatment of HAP/VAP in the ICU. Our results show significant heterogeneity in the enrollment criteria, endpoints and statistical design that may influence the ability of studies to demonstrate differences between the drugs studied. Although the methodological quality of studies seems to improve over time, some pitfalls remain. In particular, as demonstrated by the variability observed in currently unpublished RCTs testing new molecules, the regulatory agencies should agree on the best primary endpoint and the timing of its assessment. In this context, composite and/or hierarchical endpoints including both mortality and clinical cure may be of particular interest, and new methodologies helping assess the risks and benefits of new antimicrobial treatment strategies such as desirability of outcome ranking (DOOR) and response adjusted for duration of antibiotic risk (RADAR) may be used [16]. Some other promising new tools such as hierarchical nested design (combining non-inferiority and nested superiority trials) or competing event analyses (considering the influence 
of timing of events on effect measures) have also been recently proposed to improve the design and the analysis of future trials [13]. However, some basic concepts such as clinical cure remain to be better defined.

\section{Additional file}

Additional file 1: References for studies included in the systematic review. (DOCX $18 \mathrm{~kb})$

\section{Abbreviations}

APACHE: Acute Physiology and Chronic Health Evaluation; CPIS: Clinical Pulmonary Infection Score; DOOR: Desirability of outcome ranking; EMA: European Medicines Agency; FDA: Food and Drug Administration; HAP: Hospital-acquired pneumonia; ICU: Intensive care unit; ITT: Intention to treat; RADAR: Response adjusted for duration of antibiotic risk; RCT: Randomized controlled trial; SAPS: Simplified Acute Physiology Score; TOC: Test of cure; VAP: Ventilator-associated pneumonia

\section{Acknowledgements}

The authors thank Celine Feger, MD (EMIBiotech) for her editorial support.

\section{Funding}

The research leading to these results has received support from the Innovative Medicines Initiative Joint Undertaking under grant agreement number 115523 (Combatting Bacterial Resistance in Europe - COMBACTE), resources of which are composed of financial contribution from the European Union's 7th Framework Programme (FP7/2007-2013) and EFPIA companies' kind contribution.

\section{Availability of data and materials}

All data generated or analyzed during this study are included in the published articles listed in Additional file 1.

\section{Authors' contributions}

EW made substantial contributions to conception and design, or acquisition of data, or analysis and interpretation of data, and has been involved in drafting the manuscript or revising it critically for important intellectual content. WE made substantial contributions to conception and design, or acquisition of data, or analysis and interpretation of data. CA made substantial contributions to conception and design, or acquisition of data, or analysis and interpretation of data. J-RZ made substantial contributions to conception and design, or acquisition of data, or analysis and interpretation of data, and has been involved in drafting the manuscript or revising it critically for important intellectual content. J-FT made substantial contributions to conception and design, or acquisition of data, or analysis and interpretation of data, and has been involved in drafting the manuscript or revising it critically for important intellectual content. J-FT also gave final approval of the version to be published. All authors read and approved the final manuscript.

\section{Authors' information}

Not applicable.

\section{Competing interests}

The authors declare that they have no competing interests.

\section{Consent for publication}

Not applicable.

Ethics approval and consent to participate Not applicable.

\section{Publisher's Note}

Springer Nature remains neutral with regard to jurisdictional claims in published maps and institutional affiliations.

\section{Author details}

'Department of Anesthesiology and Critical Care, HUPNVS, Hôpital Beaujon, APHP, 92110 Clichy, France. ${ }^{2}$ Paris Diderot University, Paris, France. ${ }^{3}$ Inserm
UMR_S 1149 Center for Research On Inflammation Inserm/University Paris Diderot, Sorbonne Paris Cité, F-75018 Paris, France. ${ }^{4}$ UMR 1137 - IAME Team 5 - DeSCID: Decision SCiences in Infectious Diseases, control and care Inserm/University Paris Diderot, Sorbonne Paris Cité, F-75018 Paris, France. ${ }^{5}$ Physiology Department, APHP, Hôpital Cochin, Paris, France. ${ }^{6}$ Microbiology and infection control unit, APHP, Hôpital Avicenne, Paris, France. ${ }^{7}$ Medical and Infectious Intensive Care Unit, AP-HP, Hôpital Bichat Claude Bernard, Paris Diderot University, Paris F-75018, France.

Received: 13 April 2017 Accepted: 6 June 2017

Published online: 27 June 2017

\section{References}

1. Nathan C, Cars O. Antibiotic resistance-problems, progress, and prospects. N Engl J Med. 2014;371(19):1761-3.

2. Guidance for Industry Hospital-Acquired Bacterial Pneumonia and Ventilator-Associated Bacterial Pneumonia: Developing Drugs for Treatment. In: U.S. Department of Health and Human Services Food and Drug Administration Center for Drug Evaluation and Research (CDER); 2014.

3. Addendum to the guideline on the evaluation of medicinal products indicated for treatment of bacterial infections. In: European Medical Agency; 2013.

4. Richards MJ, Edwards JR, Culver DH, Gaynes RP. Nosocomial infections in medical intensive care units in the United States. National Nosocomial Infections Surveillance System. Crit Care Med. 1999;27(5):887-92.

5. Kalil AC, Metersky ML, Klompas M, Muscedere J, Sweeney DA, Palmer LB, Napolitano LM, O'Grady NP, Bartlett JG, Carratala J, et al. Management of adults with hospital-acquired and ventilator-associated pneumonia: 2016 clinical practice guidelines by the Infectious Diseases Society of America and the American Thoracic Society. Clin Infect Dis. 2016;63(5):e61-e111.

6. Bartlett JG, Barie PS, Niederman MS, Wunderink RG. Workshop on clinical trials of antibacterial agents for hospital-acquired pneumonia and ventilatorassociated pneumonia. Clin Infect Dis. 2010;51 Suppl 1:S1-3.

7. Shamseer L, Moher D, Clarke M, Ghersi D, Liberati A, Petticrew M, Shekelle P, Stewart LA, Group P-P. Preferred reporting items for systematic review and meta-analysis protocols (PRISMA-P) 2015: elaboration and explanation. BMJ. 2015;349:97647.

8. Pugin J, Auckenthaler R, Mili N, Janssens JP, Lew PD, Suter PM. Diagnosis of ventilator-associated pneumonia by bacteriologic analysis of bronchoscopic and nonbronchoscopic "blind" bronchoalveolar lavage fluid. Am Rev Respir Dis. 1991:143(5 Pt 1):1121-9.

9. Powers $\mathrm{JH}$. Recommendations for improving the design, conduct, and analysis of clinical trials in hospital-acquired pneumonia and ventilatorassociated pneumonia. Clin Infect Dis. 2010;51 Suppl 1:S18-28.

10. Klompas M. Does this patient have ventilator-associated pneumonia? JAMA. 2007:297(14):1583-93.

11. Heyland DK, Dodek P, Muscedere J, Day A, Cook D, Canadian Critical Care Trials G. Randomized trial of combination versus monotherapy for the empiric treatment of suspected ventilator-associated pneumonia. Crit Care Med. 2008:36(3):737-44.

12. Kollef MH. Review of recent clinical trials of hospital-acquired pneumonia and ventilator-associated pneumonia: a perspective from academia. Clin Infect Dis. 2010;51 Suppl 1:S29-35.

13. Timsit JF, de Kraker MEA, Sommer H, Weiss E, Bettiol E, Wolkewitz M, Nikolakopoulos S, Wilson D, Harbarth S, consortium C-N. Appropriate endpoints for evaluation of new antibiotic therapies for severe infections: a perspective from COMBACTE's STAT-Net. Intensive Care Med. 2017. doi:10. 1007/s00134-017-4802-4. Epub ahead of print.

14. Infectious Diseases Society of America, American College of Chest Physicians, American Thoracic Society, Society of Critical Care Medicine, Spellberg B, Talbot G. Recommended design features of future clinical trials of antibacterial agents for hospital-acquired bacterial pneumonia and ventilatorassociated bacterial pneumonia. Clin Infect Dis. 2010;51 Suppl 1:S150-170.

15. Sorbello AK, Komo S, Valappil T. Noninferiority margin for clinical trials of antibacterial drugs for nosocomial pneumonia. Drug Inf J. 2010;44:165-76.

16. Evans SR, Rubin D, Follmann D, Pennello G, Huskins WC, Powers JH, Schoenfeld D, Chuang-Stein C, Cosgrove SE, Fowler Jr VG, et al. Desirability of outcome ranking (DOOR) and response adjusted for duration of antibiotic risk (RADAR). Clin Infect Dis. 2015;61(5):800-6. 